\title{
Changing Paradigms in Down Syndrome: The First International Conference of the Trisomy 21 Research Society
}

\author{
Jean-Maurice Delabar ${ }^{a, b}$ Bernadette Allinquant ${ }^{c}$ Diana Bianchi ${ }^{d}$ \\ Tom Blumenthal ${ }^{\mathrm{e}}$ Alain Dekkerk Jamie Edgin ${ }^{\mathrm{f}}$ John O'Bryang \\ Mara Dierssen' Marie-Claude Potier ${ }^{a}$ Frances Wiseman ${ }^{m}$ Faycal Guedj ${ }^{d}$ \\ Nicole Créau ${ }^{a, b}$ Roger Reeves ${ }^{h} \quad K^{2}$ atheleen Gardiner ${ }^{i} \quad$ Jorge Busciglio $^{j}$

\begin{abstract}
${ }^{a}$ Brain and Spine Institute, Hospital Pitié-Salpêtrière, ${ }^{b}$ University of Paris Diderot, and ${ }^{c}$ Centre de Psychiatrie and Neurosciences, INSERM, Paris, France; ${ }^{d}$ Tufts Medical Center, Medford, Mass., e Department of Molecular, Cellular and Developmental Biology, University of Colorado, Boulder, Colo., ${ }^{\mathrm{f}}$ Department of Psychology, University of Arizona, Tucson, Ariz., 9 Department of Pharmacology, University of Illinois at Chicago, Chicago, Ill., hohn Hopkins University School of Medicine, Baltimore, Md., 'Department of Pediatrics, University of Colorado, Aurora, Colo., and ${ }^{j}$ Department of Neurobiology and Behavior, University of California-Irvine, Irvine, Calif., USA; ${ }^{\mathrm{k}}$ Department of Neurology, University Medical Center, Groningen, The Netherlands; 'Center for Genomic Regulation (CRG), Barcelona, Spain; ${ }^{\mathrm{m} I n s t i t u t e}$ of Neurology, University College, London, UK
\end{abstract}

\section{Key Words}

Down Syndrome · Trisomy 21 Research Society

\begin{abstract}
Down syndrome (DS) is the most common genetic cause of intellectual disability (ID) in humans with an incidence of $\sim 1: 1,000$ live births worldwide. It is caused by the presence of an extra copy of all or a segment of the long arm of human chromosome 21 (trisomy 21). People with DS present with a constellation of phenotypic alterations involving most organs and organ systems. ID is present in all people with DS, albeit with variable severity. DS is also the most frequent genetic cause of Alzheimer's disease (AD), and 50\% of those with DS will develop AD-related dementia. In the last few years, significant progress has been made in understanding the crucial genotype-phenotype relationships in DS, in identifying the alterations in molecular pathways
\end{abstract}

leading to the various clinical conditions present in DS, and in preclinical evaluations of potential therapies to improve the overall health and well-being of individuals with DS. In June 2015, 230 scientists, advocates, patients, and family members met in Paris for the 1st International Conference of the Trisomy 21 Research Society. Here, we report some of the most relevant presentations that took place during the meeting.

(c) 2016 S. Karger AG, Basel

The Trisomy 21 Research Society (T21RS) recently held its first meeting June 4th-7th, 2015 at the Brain and Spine Institute in the Hospital Pitié-Salpêtrière in Paris. The international conference, entitled 'Changing Paradigms in Down Syndrome', attracted 230 participants from 20 countries including scientists, clinicians, and members of associations and foundations. This report

\section{KARGER}

E-Mail karger@karger.com

www.karger.com/msy
(C) 2016 S. Karger AG, Basel

1661-8769/16/0075-0251\$39.50/0
Jorge Busciglio

BioSci III, 3216

University of California-Irvine

Irvine CA, 92697-4545 (USA)

E-Mail jbuscigl@uci.edu 
documents some of the most salient research and clinical developments presented during the meeting.

Katheleen Gardiner (USA), Roger Reeves (USA), and John Hardy (UK) delivered the initial talks that served as a comprehensive summary of the current state of knowledge regarding Down syndrome (DS). Gardiner reviewed the gene content of HSA21, emphasizing what is now known about long noncoding RNA genes and the conservation of protein-coding genes in orthologous mouse genomic regions [Gupta et al., 2016]. She also presented new data on the extensive differences in protein expression in several brain regions between male and female DS mice trisomic for the HSA21 orthologous region on mouse chromosome 10 (MMU10). The MMU10 syntenic segment encodes 4 proteins with known sex-specific functions; these must be considered candidates for causing possible sex differences in DS in phenotypic features, including responses to therapeutic interventions. Paying homage to Dr. Muriel Davisson, Roger Reeves reviewed the history of the Ts65Dn, the first viable segmental trisomy mouse model of DS, and the inestimable value of its role in furthering DS research. He also reviewed his research on cerebellar abnormalities in the Ts65Dn, the role of sonic hedgehog, and the use of zebrafish to identify candidate causative genes. He further described initial comparison of the Ts65Dn features with those of the newer Dp(16)1Yey DS mouse model which is trisomic for the entirety of the MMU16/HSA21 syntenic region. Dr. Hardy reviewed efforts to study the genetics of Alzheimer's disease (AD) in DS, touching on the concept that all individuals with $\mathrm{DS}$ indeed develop $\mathrm{AD}$-related dementia, the role of ApoE4 allelic variation, and describing the LonDownS consortium which is collecting samples from individuals with DS at multiple ages and carrying out SNP analysis.

\section{Neuropathology in the Adult with DS}

Life expectancy and quality of life for individuals with DS have dramatically improved in recent years. However, with increasing age, pathological dysfunctions are exacerbated, including the development of AD neuropathology in nearly all individuals with DS and dementia in almost half of individuals with DS age 50 and older.

Elizabeth Head (USA) focused on white matter integrity and cerebrovascular pathology in older individuals with DS as part of a longitudinal study of aging and dementia. Using diffusion tensor imaging, Head and colleagues showed that frontal white matter integrity is sig- nificantly lower in DS compared to non-DS controls and is further compromised in DS dementia and correlated with neuropsychological test scores. The results linked white matter loss to vasculature changes and/or neuroinflammation in DS, both of which are under current investigation.

Stanley Durrleman (France) presented an alternative approach for the analysis of variations of the neuroanatomical phenotype by specifically analyzing the shape of deep brain structures. This approach relies on the estimation ofoneor several referenceanatomical configuration(s) specific to a group of individuals. The anatomical configuration of each subject is compared to the reference(s) via spatial deformations. This method allows interpretation of differences between individuals or between populations as typical deformations of their brain structures.

Besides the anatomical shape, understanding the role and importance of connectivity patterns is crucial. Pathologies in intellectual disabilities present with characteristic alterations in neuronal structure and connectivity leading to changes in neuronal network dynamics and function. To systematically analyze short- and long-range connectivity patterns, Mara Dierssen (Spain) presented examples in mouse models of DS (Ts65Dn and TgDYRK1A) that recapitulate both cognitive impairment and morphological alterations observed in DS.

The deregulation of broad protein networks driven by a broad transcriptional deregulation as a consequence of trisomy 21 is a field of intense research. Valerie Hindie (France) presented a DS-related protein interaction map generated by using high-throughput, domain-based yeast 2 -hybrid technology. Fifty-two human proteins, selected for their putative role in DS and associated intellectual deficiency, were used as entry points to screen a randomprimed adult brain cDNA library. More than 1,200 protein-protein interactions were identified and integrated with protein interaction data from the literature. This will eventually make the creation of a comprehensive interaction map centered on DS proteins possible.

By analyzing brain tissue from DS patients with and without AD, Marzia Perluigi (Italy) showed that pathological hallmarks common to $\mathrm{DS}$ and $\mathrm{AD}$, which include plaque deposition, neurofibrillary tangles, oxidative damage, and impaired mitochondrial function, are all related to oxidative stress. While a low amount of oxidative stress can activate protective cellular mechanisms, such as the antioxidant and heat-shock response, cell cycle regulation, DNA repair as well as unfolded protein response and autophagy, chronic oxidative stress causes irreversible damage to intracellular macromolecules including 
protein oxidation. Accumulation of damaged proteins, increasingly prone to aggregation, appears to be a key factor leading to neuronal death.

Finally, Cynthia Lemere (USA) presented evidence that, although the onset of AD pathogenesis varies in $\mathrm{DS}$ brains, intracellular amyloid beta $(\mathrm{A} \beta) 42$ precedes deposition of extracellular diffuse $A \beta 42$ plaques, which is followed by plaque maturation, neuritic dystrophy, inflammation, vascular amyloid, and the formation of neurofibrillary tangles in the neocortex. It is still unclear which factors determine the timing of the onset of AD pathology in DS or the tipping point for clinical dementia.

\section{Beyond Neuropathology and Cognition}

John O'Bryan (USA) discussed the role of the intersectin 1 (ITSN1) scaffold protein in DS-associated malignancies. ITSN1 is overexpressed in DS patients, and the O'Bryan group previously demonstrated that ITSN1 activates the Ras proto oncogene on endocytic vesicles. $\mathrm{He}$ presented data on the fabrication and properties of a novel, protein-based inhibitor of Ras. This engineered protein selectively bound Ras and inhibited its ability to activate downstream signaling pathways such as the ERKMAPK. In addition, this Ras inhibitor blocked Ras-mediated transformation. O'Bryan also discussed potential mechanisms by which this engineered protein inhibited Ras function and how such inhibitors may be relevant to DS-associated cancers:

S. Ogawa (Japan) presented an analysis of genomic mutations present in DS-acute megakaryoblastic leukemia (DS-AMKL). While DS patients exhibit a reduced incidence of solid tumors, they have an elevated risk for leukemias. Approximately 5\% of DS children develop a transient myeloproliferative disorder (TAM) at birth. All of these patients possess mutations in the GATA1 transcription factor. While this TAM resolves in most cases, roughly $20 \%$ progress to DS-AMKL. Ogawa has identified additional mutations present in DS-AMKL, but not TAM, in components of the cohesion complex (STAG2, $R A D 21, S M C 1 A$, and SMC3). Genetic modeling of these mutations in mice revealed that loss of STAG2 increased myeloid proliferation, and conditional deletion of SMC3 increased the hematopoietic stem cell and progenitor populations.

John Crispino (USA) presented work on the dualspecificity tyrosine regulated kinase DYRK1A in normal and malignant hematopoiesis. His laboratory previously described a tumor-promoting role for DYRK1A in DS-
AMKL, although its role in normal hematopoiesis was unclear. Here, he showed that loss of DYRK1A resulted in defects in both B-and T-cell lineages without affecting myeloid development using conditional DYRK1A mouse models. These results were mimicked by pharmacological inhibition of DYRK1A with EHT-1610. Conditional DYRK1A mouse model lymphoid cells failed to exit the cell cycle and exhibited defects in DREAM targets with increases in phospho-Rb, cyclin D2 and cyclin D3. Given the high expression of DYRK1A in B- and T- lineage ALL, they tested the efficacy of DYRK1A inhibition and found that EHT-1610 inhibited the growth of both B-ALL and T-ALL suggesting DYRK1A inhibitors may be efficacious in these malignancies.

Michel Polak (France) presented results on the role of DYRK1A in thyroid dysfunction in DS. His laboratory found that a BAC transgenic DYRK1A mouse model with 3 copies of DYRK1A exhibited a decrease in the thyroglobulin-positive surface area, a decrease in T4 levels and an increase in TSH levels compared to wild-type control mice. The thyroid showed the same degree of abnormal morphogenesis as observed in human fetuses with DS. Although DYRK1A was sufficient for this hypothyroid phenotype, it was not clear whether DYRK1A was necessary for this thyroid dysfunction in the context of any mouse model of DS, such as Ts65DN or Ts1Cje.

Latif Rachdi (France) presented another vignette on DYRK1A but in the context of pancreatic $\beta$-cell function. DS children have $\sim 4$-fold increased risk for type 1 diabetes mellitus. Using a combination of mouse models, they demonstrated that haploinsufficiency of DYRK1A induces diabetes through decreasing $\beta$-cell mass. In contrast, Dyrk1A overexpression in mBACTg DYRK1A mice results in an increased $\beta$-cell mass. These latter mice were lean and resistant to high-fat diet-induced $\beta$-cell failure. DYRK1A overexpression appeared to function through increasing the pancreatic progenitor population.

Damien Keating (Australia) talked about the identification of genes involved in insulin secretion and diabetes. As discussed previously by Rachdi, diabetes is more prevalent in DS patients. By comparing the glycemic status of different DS mouse models, they identified a region in chromosome 21 containing 38 genes that contribute to hyperglycemia and poorer glucose tolerance. $R C A N 1$ was identified as the lead candidate gene. $R C A N 1$ overexpression in mice resulted in decreased circulating insulin levels, $\beta$-cell area and number, and $\beta$ proliferation. Furthermore, the remaining $\beta$-cells display decreased glucose-stimulated insulin secretion. The involvement of RCAN1 in insulin secretion appears to be linked to mitochondrial function. 
Eva Lana-Elola (UK) introduced the audience to the study of atrioventricular septal defects, commonly present in DS. To identify the genomic region(s) that contain genes causing these congenital heart defects, Lana-Elola and coworkers generated a new panel of mouse lines that are trisomic for subsets of HSA21-orthologs in MMU16. She demonstrated that atrioventricular septal defects are caused by a third copy of at least 2 different genes.

\section{Biomarkers of Pathology Progression in DS}

Yann Hérault (France) discussed new models of DS with segmental duplication including regions of MMU17 (dup17 or Ts1Yah) orthologous to HSA21. Mouse models allow identification of deregulated pathways for further studies on therapeutic interventions and the selection of biomarkers. The Ts1Yah mice show recognition memory deficits that can be compensated by either decreasing the number of copies of the Cbs gene from 3 to 2 or by increasing $D Y R K 1 A$, thus highlighting interactions between these 2 crucial genes, Cbs and DYRK1A.

Elliot Mufson (USA) showed that AD pathology in DS and in non-DS AD shows similarities, including decreased numbers of magnocellular cholinergic neurons, decreased levels of TrkA in temporal cortex, and increased levels of proNGF and MMP9. There were, however, marked differences in the number of thioflavin-positive neurons and the distribution of tangles with close proximity to amyloid plaques and dystrophic neuritis, suggesting that DS neurons generate a different response to the amyloid load.

Fabian Fernandez (USA) presented new circadian biomarkers in DS. He studied 2 to 4 -year-old individuals with DS who exhibit weaker circadian rhythm activities as compared to typically developing children. DS is associated with decreased intraday activity consolidation and decreased ultradian rhythm patterns of behavioral activity.

Nathalie Janel (France) proposed DYRK1A as a new plasma biomarker in DS and AD. Indeed, DYRK1A was found in plasma and its level is correlated to the number of copies of the corresponding gene. Interestingly, 2 independent cohorts of $\mathrm{AD}$ patients showed a significant decrease of DYRK1A plasma levels during progression of the disease which correlated to the amyloid load in the brain and cognitive impairment.

Peter Paul De Deyn (Antwerp, Belgium) showed that the level of biogenic amines in plasma of DS individuals correlates with the behavioral and psychological symp- toms of dementia. In particular, 3-methoxy-4-hydroxyphenylglycol appeared to predict the conversion to AD dementia in those with DS.

Florencia Iulita (Canada) expanded on the potential of plasma $A \beta$ as a biomarker for AD in DS. In her study, plasma levels of both $A \beta 40$ and $A \beta 42$ were elevated in DS individuals with dementia and negatively correlated with cognitive decline in asymptomatic subjects without dementia.

\section{Prenatal Diagnosis and Treatment of DS}

Tarik Haydar (USA) reported on developmental brain abnormalities in human fetuses and in the Ts65Dn and Ts1Cje mouse models [Haydar and Reeves, 2012], including a longer neurocortical cell cycle, reduced neurogenesis, microcephaly, and hypocellularity in the neocortex, cerebellum and hippocampus. He also described recent experiments that unexpectedly showed that the Dp (16) $1 \mathrm{Yey} /{ }^{+}$mouse model does not have prenatal abnormalities in forebrain growth or morphology, nor reduced neurogenesis or abnormal mitotic activity. Along those lines, behavioral abnormalities in this model are not observed until 2-3 weeks of postnatal age.

Tomo Tarui (USA) reviewed DS fetal neuropathology, which is characterized by microcephaly, overall decreased cell numbers as well as decreased cell density and proliferation in the second trimester cerebellum and hippocampus. Other prenatal findings in DS include disorganized lamination and abnormal neuronal differentiation, accompanied by a decreased presence of neurotransmitters in the frontal lobe. Abnormal prenatal sonographic findings include decreased frontothalamic distances and transcerebellar diameters. Tarui also presented preliminary data from his MRI study showing that cerebellar growth lagged in fetal brains from fetuses with DS.

Diana Bianchi (USA) reported on her functional genomic analysis of amniotic fluid supernatant cell-free RNA to identify genes that are differentially expressed in living human fetuses with trisomy 21 compared to ageand sex-matched fetuses with a normal chromosome number [Slonim et al., 2009]. Preliminary results from an initial study of apigenin, a natural antioxidant and antiinflammatory compound found in citrus fruits and leafy green vegetables, given at the time of conception and throughout pregnancy to wild-type C57Bl/6 dams mated to Ts1Cje males [Guedj et al., 2014] indicate that apigenin improved the time frame of achieving developmental milestones for Ts1Cje pups. 
Renata Bartesaghi (Italy) explained the rationale for using fluoxetine as a treatment for DS. The serotonergic system is important for neurogenesis and dendritic development, and it is altered in DS. She and her colleagues gave fluoxetine to pregnant Ts65Dn mice from embryonic day 10 to birth to inhibit serotonin re-uptake and examined short- (P2) and long-term (P45) outcomes [Stagni et al., 2013; Guidi et al., 2014]. Fluoxetine restored overall cellularity and connectivity in multiple brain locations. This effect persisted long-term following treatment cessation and was accompanied by significant prevention of cognitive deficits.

Jean-Maurice Delabar's (France) group focused on ameliorating changes in the expression of the HSA21 kinase, Dyrkla, which contributes to the control of excitation-inhibition balance [Guedj et al., 2009; Souchet et al., 2014]. They compared 3 different extracts of green tea, the properties of which include inhibition of Dyrkla, and showed that in mBACtgDyrkla and the Dp(16)1Yey/ ${ }^{+}$ transgenic mice, the deficits in Y maze performance were corrected by treatment with $60 \mathrm{mg} / \mathrm{kg} /$ day of green tea extract. They also tested continuous treatment of pregnant mothers throughout gestation until weaning and pups until 3 months after birth. When the treatment was stopped at weaning, the effects on density of GAD67 neurons and excitation/inhibition balance persisted past the washout phase of the nutritional supplement.

Jessica Ash (UK) presented her study demonstrating the beneficial effects of maternal choline supplementation (MCS) in the Ts65Dn mouse. Choline is an essential nutrient during fetal development due to its role in acetyl choline production, cellular membrane formation, and epigenetic regulation. MCS produced a dramatic improvement in spatial cognition in aged Ts65Dn offspring, which correlated with hippocampal neurogenesis [Velazquez et al., 2013] as well as increased density and size of basal forebrain cholinergic neurons [Ash et al., 2014]. MCS also improved attention and emotional regulation in aged Ts65Dn mice [Moon et al., 2010].

In summary, several talks made a strong case for the prenatal period as being one of potentially several 'windows of opportunity' for the treatment of DS. The availability of a prenatal treatment option for pregnant women carrying fetuses with DS would significantly change the current paradigms of prenatal care [Bianchi, 2012; Guedj and Bianchi, 2013]. However, fetal development is obviously a sensitive time period, and the potential adverse effects of treatments must be considered carefully. For example, fluoxetine is not even approved for use in children.

Changing Paradigms in Down Syndrome

\section{From Early Development to Ageing in DS}

Two breakthrough sessions covered recent results related to disease phenotypes affecting DS individuals across the life span. Prachi Patkee (UK) showed preliminary MRI data indicating decreased volumes of the whole brain, and specifically cortex and cerebellum, in DS fetuses that were associated with enlargement of the fourth ventricle.

Margherita Scarpato (Italy) studied global gene deregulation in DS embryos, with the hypothesis that overexpression of chromosome 21 genes may perturb the physiological regulatory mRNA-miRNA network. Computational analyses selected the HUNK kinase to be the strongest HSA21 candidate gene for the regulation of expression of mRNAs, and experimental studies have confirmed that overexpression of the HUNK $3^{\prime}$ UTR induces an increase in expression of genes involved in embryonic stages and decreases corresponding miRNAs. This dysregulation of the mRNA-miRNAs network may be a partial explanation for the multisystem perturbations occurring in DS. Abnormal protein maturation has been observed in the DS brain. This is the case for the nerve growth factor (NGF), where levels are decreased in the brains of $\mathrm{AD}$ and DS patients. Claudio Cuello (Canada) reported a significant increase in levels of proNGF and decreases in levels of plasminogen and tPA, which are involved in the maturation of proNGF. In parallel, mature NGF has a decreased half-life due to elevated activity of MMP9, the major NGF-degrading protease. Consequently, resulting decreased levels of mature NGF would contribute to the observed atrophy of basal forebrain cholinergic neurons. Alterations in proNGF and MMP9 were also observed in DS neurons in culture, suggesting that the defect in NGF trophic support is an early event in DS.

Dimitry Goldbager (USA) proposed that anomalies in embryogenesis in DS might be related to a failure in the mechanism of communication between embryonic and maternal tissues. The pericentrin gene, involved in meiosis, mitosis and ciliogenesis, is a candidate gene for these communications. Mutations in the pericentrin gene cause dwarfism and ciliopathies. Variants of this gene in DS have not yet been studied.

Alexander Kleschevnikov (USA) focused on mechanisms of altered brain functioning in the Ts65Dn DS model. Specifically, the evaluation of the role of the trisomy of the $\mathrm{G}$ protein regulated inwardly rectifying potassium channel KCNJ6 (GIRK2, KIR3.2), by crossing Ts65Dn and Kcnj6KO mice to reduce KCNJ6 copy num- 
ber. This intervention normalized hippocampal longterm potentiation and some behavioral phenotypes (longterm object recognition memory vs. working memory).

During development, the brain chloride gradient is important to drive GABA from excitatory to inhibitory, and it is associated to seizure vulnerability. This was Andrea Contestabile's (Italy) topic. After birth in the normal brain, the decrease in NKCC1 $\left(\mathrm{Na}^{+}-\mathrm{K}^{+}-\mathrm{Cl}^{-} 1\right.$ cotransporter) and increase in $\mathrm{KCC} 2\left(\mathrm{~K}^{+}-\mathrm{Cl}^{-}\right.$cotransporter) result in inhibitory GABA. In the hippocampal CA1-CA3 of adult Ts65Dn, the potential was found depolarizing, resulting in GABA excitation as a result of increased NKCC1 protein. Acute or long-term treatments of Ts$65 \mathrm{Dn}$ mice with bumetanide, an inhibitor of NKCC1, restored the inhibition and their defects in the novel-object recognition test [Deidda et al., 2015]. Alterations in the excitation/inhibition balance due to increased GABAergic signaling have been previously shown in the brain of DS models. These new data identify a novel therapeutic approach to rescue cognitive abilities in DS.

The incidence of fractures is elevated in adults with DS resulting from osteoporosis and osteopenia. To evaluate these phenotypes in mouse models, Randall Roper (USA) examined trabecular bone mineral density, cortical width, and biomechanical parameters of the femur in Ts65Dn males. The decreases in mineralization, cortical width, bone stiffness, and ultimate load together with reduced osteoclasts number were rescued by reducing DYRK1A copy number (Ts65Dn $\left.\times D Y R K 1 A^{+/-}\right)$. Thus, the osteopenic phenotype associated with DS may be rescued postnatally by targeting trisomic DYRK1A.

Finally, Eugene Yu (USA) presented the study of the mouse model trisomic for all 3 mouse syntenic regions

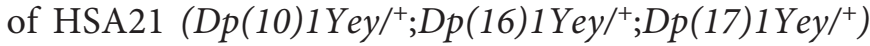
to evaluate the role of trisomy in the mechanisms of myeloproliferative disorder and DS-AMKL. The complete trisomy induced splenomegaly and an increase in megakaryocyte progenitors related to a MMU16 region common in $\mathrm{Dp}(16) \mathrm{Ye}^{+}$and Ts65Dn. Interestingly, adding a GATA1 mutation amplified the effect of the trisomy leading to a more severe macrocytic anemia.

\section{Defining Translational Pathways for DS}

Susana de la Luna (Spain) reviewed the properties of $D Y R K 1 A$, a kinase and the most popular gene located in chromosome 21. In particular, several neurological phenotypes are observed in individuals with reduced expression of DYRK1A, an important fact to consider when pro- posing the use of DYRK1A inhibitors in a clinical setting. She then discussed some of the many nuclear and cytoplasmic targets of this kinase, including RNA polymerase II [Di Vona et al., 2015].

Weihong Song (Canada) reviewed the role of the enzymes BACE1, the $\beta$-secretase that cleaves the amyloid precursor protein (APP) to generate $\mathrm{A} \beta$ [Zhang et al., 2015] and BACE2, a chromosome 21 encoded paralogue of BACE1, which may prevent the formation of $A \beta$ [Sun et al., 2006]. The results showed that the chromosome 21 gene RCAN1 modulates the level of APP via a differential effect on proteasome activity [Wu et al., 2015].

Frances Wiseman (UK) presented the results of her work directed at understanding the role of HSA21 genes other than APP in the development of AD pathology and dementia in DS. She uses the Tc1 mouse model of DS which is trisomic for $75 \%$ of chromosome 21 genes but not APP [O'Doherty et al., 2005; Gribble et al., 2013]. Her results suggest that the trisomy of genes other than $A P P$ can exacerbate $A \beta$ aggregation and deposition, and the associated cognitive deficits seen in a common mutant human APP transgenic mouse model.

Ann-Charlotte Granholm-Bentley (USA) continued the theme of mechanisms in AD and DS presenting evidence of the effects of inflammation on cognitive decline in the Ts65Dn mouse model of DS [Hunter et al., 2004] and showing that failure of the normal resolution of the inflammatory response contributes to disease progression and may be a target for treatment.

Marie-Claude Potier (France) talked about her studies of the endosome-lysosome defects associated with overexpression of the chromosome 21 gene SYNJ1 which causes endosomal enlargement [Cossec et al., 2012]. Additionally, upregulation of cholesterol in cellular membranes can also cause endosomal enlargement, leading to alterations in APP trafficking and processing [Marquer et al., 2014].

William Mobley (USA) finished the session with an overview of $\mathrm{AD}$ pathological processes that may be amenable to treatment in people with DS including the key role of APP C-terminal fragments, particularly of $\beta$-CTF in Rab5 activation leading to failure of endosomal transport [Salehi et al., 2016].

\section{Phenotypic Studies in DS - Animal and Cellular Models}

Two presentations reported efforts to identify candidate therapeutic interventions, 1 for the prenatal period and 1 for aging. Takashi Minami (Japan) used a condi- 
tional $D s c r 1^{-/-}$mouse model and showed abnormal vascular branch formation in several organs, including the brain, and that ApoE protected $D s c r 1^{-/-}$mice against atherosclerotic plaques in the aorta. The relationship of this approach to therapy for DS is unclear. Heather McGowan (USA) used induced pluripotent stem cells (iPSCs) and associated induced neurons (iNs) to study the target genes of the 5 HSA2 1 miRNAs; she found that MeCP2 expression is downregulated in iPSCs and iNs in individuals with DS. Antagonizing HSA21 miRNAs through 'Tough Decoy' inhibitors normalized iPSCs and iNs $\mathrm{MeCP} 2$ expression and synaptic function. Alexandra Botté (France) showed that early endosomes are enlarged, form aggregates, and their number as well as the number of lysosomes are increased in cholinergic neurons of adult Ts65Dn mice. Notably, the Ts65Dn is trisomic for both SYNJ1 (HSA21 ortholog) and SYNJ2 (HSA6 ortholog; see above, regarding SYNJ1 in endosome function and properties). Faycal Guedj (USA) tested the effects of prenatal treatment with apigenin on fetal brain gene expression and behavior in the Ts1Cje mouse model of DS which is trisomic for $\sim 40 \%$ of HSA21 orthologs. Apigenin (400 $\mathrm{mg} / \mathrm{kg} /$ day) rescued some features of gene expression as well as neonatal and adult behavior. Eric Hamlett (USA) demonstrated that a one-month treatment of adult Ts65Dn mice with Resolvin E1 (5 $\mu \mathrm{g} / \mathrm{kg} /$ day), a bioactive pro-resolution ligand, enhanced memory performance in the radial arm water maze and novel object recognition tests. This improvement was accompanied by a significant reduction of both inflammatory cytokine levels and microglia activation.

\section{Behavioral Correlates in Animal Models of DS}

Marta Fructuoso (Spain) presented her study of feeding behavior, glucose homeostasis, and endocrine pancreatic functioning in the Ts65Dn mouse model of DS. Young Ts65Dn mice on standard chow diet had an increased average food intake and became obese when exposed to a high-fat diet, which reflects the higher prevalence of obesity within the DS population.

Pratiksha Rao (India) focused on the response time during a dual motor task among children with intellectual disabilities. Children with DS were the slowest and had the least force during the task when compared to children with intellectual disabilities of another etiology or typically developing children.

Subsequently, Alain Dekker (The Netherlands) elaborated on behavioral and psychological symptoms of de- mentia (BPSD) in DS. He stressed that particular BPSD are often present long before the clinical diagnosis of dementia and may thus serve as early indicators for $\mathrm{AD}$ in DS. To accurately assess BPSD in DS, and thus enable adaptive caregiving and early therapeutic interventions, Dekker and colleagues developed the novel and all-embracing BPSD-DS evaluation scale.

Thu Lan Nguyen (France) presented her data on behavioral and cognitive improvement in mice that overexpressed Dyrkla either alone or in combination with additional HSA2 1 orthologs after treatment with Leucettine 41 , a specific synthetic DYRK1A inhibitor.

\section{Cognition in DS}

Annette Karmiloff-Smith (USA) emphasized that endpoint outcomes evolve across development, and therefore, to really understand the evolution of a disorder such as $\mathrm{AD}$ in $\mathrm{DS}$, we need to track the progression of the disease from infancy. Her work, using cutting-edge methods of eye tracking, demonstrates impairments in attention and memory formation as early as 6 months in DS. She also discussed the importance of understanding how impairments in basic processes, such as attention and sleep, may impact development in other areas, e.g., language.

Pamela Banta-Lavenex (Switzerland) presented her work teasing apart the spatial navigation skills of older children and young adults with DS, finding that this group showed intact egocentric search but deficits in allocentric search, reflecting the presence of hippocampal deficits in DS. Of interest were the considerable individual differences in search patterns, suggesting variation in the development of the hippocampal formation. Very few participants with DS were capable of the precision searches that require intact hippocampal development.

In a talk focused on language, Len Abbeduto (USA) presented data on a study of expressive language sampling conducted across 5 sites in the United States. A conversation sample, picture book narration, and $\mathrm{Au}-$ tism Diagnostic Observation Schedule were administered to all children to assess practice effects and to measure retest reliability and concurrent validity with other commonly used measures. Expressive language sampling measures had excellent test-retest reliability and good concurrent validity, but practice effects were found on the conversation task. This consortium will be one of the largest DS cohorts studied for language development to date. 
Jamie Edgin (USA) presented her data on correlations between sleep and cognitive development, showing strong relations between obstructive sleep apnea and reduced expressive language as well as executive function in toddlers and school-age children with DS. Data suggested that sleep disturbances in DS are not adequately measured through parent report and that objective measures are required. Future studies will examine the timing of the emergence of these deficits in infancy and the mechanisms underlying the links between sleep disruptions and learning deficits. Nancy Lee (USA) presented her work on measurements of the cortical surface area and thickness collected in a large sample of children with DS. While she found overall grey matter reductions, these reductions stemmed from regional differences in the surface area, not reductions in cortical thickness, which, conversely, was thicker on average. Surface area reductions were most prominent in the frontal and temporal lobes.

Debbie Fidler (USA) expanded our understanding of deficits in executive function in DS, which were consistently measured on a number of assessments in early childhood. Nonverbal rather than verbal IQ predicted the extent of executive deficits. Given the importance of these skills for the development in a number of cognitive domains, more work needs to be done to translate this aspect of the phenotype into targets of behavioral and pharmacological intervention starting early in development.

\section{Impact and Strategies for Aneuploidy in Gene Expression and Metabolism}

The triplication of HSA21 in DS leads to an increase in gene transcription. To restore the expression of particular genes, Marianne Rots (The Netherlands) induced gene reprogramming by rewriting epigenetic marks at a genomic locus of interest (epigenetic editing). This was achieved by engineering DNA-binding zinc finger proteins or by employing CRISPR/dCas9 fused to epigenetic writers/erasers to locally rewrite the epigenetic signature. The results successfully demonstrated that rewriting epigenetic marks reprograms gene expression, thus offering possibly novel approaches to restore disturbed gene expression profiles.

Ralph Nixon (USA) presented compelling evidence that APPL1 (adaptor protein containing pleckstrin homology domain, phosphotyrosine-binding domain and leucine zipper motif) mediates the overactivation of the early endosomal regulator, Rab5 in DS and AD. This is caused by increased levels of the $\beta$-cleaved carboxy-ter- minal fragment of APP ( $\beta \mathrm{CTF})$, which recruits APPL1 to Rab5 endosomes, leading to accelerated endocytosis, endosome swelling, and impaired axonal transport of Rab5containing endosomes. These effects were corrected in DS fibroblasts by APPL1 knockdown. $\beta$ CTF levels were also found elevated in AD brains. These results show that overactivation of Rab5 through $\beta$ CTF-APPL1 interactions constitutes a novel APP-dependent pathogenic pathway in $\mathrm{DS}$ and $\mathrm{AD}$.

Jorge Busciglio (USA) discussed recent findings implicating an impaired mitochondrial metabolism as a key factor leading to common abnormalities in cellular function in 3 different human aneuploidies (trisomies 13, 18, and 21). Mitochondrial-targeted expression of the antioxidant enzyme catalase ameliorated both mitochondrial parameters and cellular function as measured by in vitro wound healing assays. The results provide further evidence supporting the implementation of therapeutic strategies to preserve mitochondrial function and prevent oxidative stress in DS.

Reinier Veitia (France) discussed the molecular and cellular effects of gene dosage imbalances, which can lead to abnormal phenotypes when occurring within macromolecular complexes and cellular circuits. Potential therapeutic approaches to 'buffer' or attenuate the abnormal phenotypes include stimulation of mRNA or protein degradation (pharmacological modulation of proteolysis), which may help to palliate the deleterious effects of aneuploidy in cellular homeostasis.

\section{Clinical Evaluation and Caring in DS}

David Stein (USA) searched for factors that are associated with inclusion in the standard classroom because it has been shown to improve cognitive development in children with DS. Sixty-three consecutively referred children with DS underwent neuropsychological testing in an urban hospital setting. IQ, language, social skills, and adaptive function were examined as well as family socioeconomic status. Of these possible indicators, only the socioeconomic status powerfully predicted inclusion, suggesting that the individual needs of children did not determine the inclusion status in the sample.

Obstructive sleep apnea is prominent in people with DS with a prevalence ranging between $55-97 \%$, compared to $1-4 \%$ in the typical pediatric population. The objective of Brian Skotko's (USA) study was to construct a clinically useful screening tool for obstructive sleep apnea by using a combination of signs, symptoms, cranio- 
facial anatomical measurements, and metabolic markers. They enrolled 130 patients with DS (ages 3-24 years) as an observational, prospective cohort study. The significant variables included results of survey questions, medication history, anthropometric measurements, vital signs, patient's age, and physical examination findings. With $90 \%$ accuracy, a negative result with their model predicted that the person does not have moderate to severe obstructive sleep apnea. Because of the false positive rate, results need to be confirmed with a sleep study.

\section{Assessment of Therapeutic Approaches and Clinical Trials for DS}

Maddalena Adorno (USA) reported that USP16, a HSA21-encoded protein, deubiquinates histone $\mathrm{H} 2 \mathrm{~A}$, a critical mark for the maintenance of multiple somatic tissues. An increased level of this protein is associated with accelerated senescence of Ts65Dn fibroblasts. Downregulation using a short interfering RNA rescued abnormalities in stem cell properties and defects in proliferation of Ts65Dn and DS fibroblasts [Adorno et al., 2013]. Because of its role in controlling senescence pathways, USP16 is an attractive target to potentially ameliorate some of DS pathologies.

Andre Strydom (UK) described the presentation of dementia in DS, which differs from typical AD [Sheehan et al., 2014]. He compared criteria used in the International Classification of Diseases (ICD-10), the Diagnostic and Statistical Manual of Mental Disorders (DSM-IV-TR) dementia criteria, and the DSM-5 for neurocognitive disorder. He presented data from the LondownS consortium on the floor and ceiling effects of cognitive tests in DS and concluded that standardization of dementia diagnoses is necessary for future trials in older adults.

Michael Raffi (USA) presented the DS Biomarker Initiative project which started with a pilot study (3 years) on DS individuals without dementia (30-60 years old). Participants underwent extensive cognitive testing and imaging. Most subjects demonstrated amyloid PET positivity reflecting fibrillar amyloid plaque deposition. Results from multimodal analysis suggest a greater hippocampal atrophy with amyloid load. Cognitive and functional measures did not correlate with amyloid load in DS but did correlate with regional FDG PET measures. He concluded by saying that biomarkers of $\mathrm{AD}$ can be readily studied in adults with DS and that a multicenter longitudinal study should be performed to understand the trajectories of AD markers in DS [Ness et al., 2012].

Changing Paradigms in Down Syndrome
Clothilde Mircher (France) started her presentation by highlighting the crucial role of the thyroid hormone for brain development and maturation as well as the consequences of a lack of folates in malformation and neurocognitive disorders. She also mentioned previous results of 'ENTRAIN', a study suggesting a possible synergy between folates supplementation and thyroxine [Blehaut et al., 2010]. She presented 'ACTHYF' a monocentric, random, comparative phase III study, in 4 arms (folinic acid, thyroid hormone, both, neither one). At inclusion, patient age ranged from 6 to 18 months. The design includes a primary and secondary end point, biometry and biological markers.

Jeannie Visootsak (USA) described a clinical trial based upon observations of an excessive GABAA inhibitory signal in one mice model of DS. She described the properties of the drug RG1662 that targets the alpha 5 subunit. She continued her presentation by describing a safety study on healthy adults and DS as well as a study for assessing cognitive tests in teens and adults with DS [Visootsak et al., 2011]. Furthermore, she presented 2 phase II studies: one on a group of 12-30 years and the other on a group of 6-11 years. Both multicentric studies will use composite primary end points to demonstrate clinically meaningful outcomes.

Rafael de la Torre (Spain) presented the third clinical trial of this session: he described the rationale to use a DYRK1A inhibitor [Guedj et al., 2009] - namely, EGCG treatment in DS. He referred to results of a pilot study using a green tea extract on young adults with DS: safety of extract, effects in memory tests, which vanished after discontinuing the treatments. Following, he described the TESDAD study ( 85 individuals, $18-30$ years old), a phase II clinical trial, double-balanced placebo design: placebo and active treatment condition encompass cognitive stimulation. Endpoints included a cognitive test battery [de Sola et al., 2015], imaging [Pujol et al., 2015], and biomarkers [Noll et al., 2009]. The clinical global index shows an effect of the treatment, maintained 6 months after interruption of the treatment, which also rescued normal levels of biomarkers and restored normal functional connectivity.

\section{T21RS: A Link between Science and Society}

Besides being the scientific network for the DS research community, T21RS aims to be a source of scientifically founded information. This is why the Committee for Science \& Society has been established, led by Peter 
Paul De Deyn (Antwerp, Belgium). The Committee provides national and international DS associations with updates on recent scientific discoveries via the T21RS Science \& Society Bulletin and also encourages these associations to give feedback and point at issues that advocates and participants feel should be investigated. The special T21RS Science \& Society Symposium was organized on Sunday, June 7 th, to (1) more extensively introduce T21RS and the Committee for Science \& Society to representatives of these associations, (2) get acquainted with each other, (3) discuss the gap between science and society, and (4) share thoughts and ideas about future directions.

Alain Dekker (Groningen, The Netherlands) commenced with a brief introduction to T21RS and looked back upon the first year of the society's existence, including its establishment, organizational structure, and the latest details on the number of members and their spreading over the globe. In the subsequent association introduction round, 13 associations shortly pitched their main goals, initiatives, and perspective in 3 minutes: (1) Alzheimer's Association ISTAART Professional Interest Area (PIA): DS-AD (Cindy Lemere, USA), (2) LuMind RDS Foundation (Michael Harpold, USA), (3) The Matthew Foundation (John Blascovich and Maddalena Adorno, USA), (4) Global Down Syndrome Foundation (Michelle Sie Whitten, USA), (5) Fondation Jérome Lejeune (Thierry de la Villejegu, France), (6) Trisomie 21 France (Cécile Dupas, France), (7) Down Syndrome International (Andrew Boys, UK), (8a) Down España and (8b) European Down Syndrome Association (both represented by Manuel Velázquez López, Spain), (9) Fondation AMIPIBernard Vendre (Remi Cornubert and Marie-Laure Blandin, France), (10) Catalan Down Syndrome Foundation (Sebastián Videla, Spain), (11) Association Française pour la Recherche sur la Trisomie 21 (Agnes Duguet, France), and (12) Hungarian Down Association (Hungary).
Next, Peter Paul De Deyn elaborated on the achievements and initiatives of the T21RS Committee for Science \& Society. One initiative in which the Committee is involved is The Dementia Table Initiative in The Netherlands. Sandra Overbeek and Charlotte Toolen (Utrecht, The Netherlands) presented their work to set up special evening gatherings, so-called Dementia Tables, in the 12 provinces of The Netherlands, where relatives and caregivers can share experiences and improve knowledge about ageing and dementia in people with intellectual disabilities. They expanded on the important aspects of the organization and gave tools for initiating such sessions in other countries. The positive responses and questions from the audience opened the general discussion about Science \& Society, which specifically focused on closing the gap between science and society as well as strengthening and expanding collaborations between the Committee and the DS associations.

In summary, the International Conference of the T21RS is poised to become a very important and regular international forum for scientists, clinicians, and advocates actively working to improve the quality of life of people with DS. Communication and cooperation is the key to continuing the truly remarkable progress in a condition that was deemed as 'untreatable' just a few years ago.

\section{Acknowledgments}

The 1st International Conference of the Trisomy 21 Research Society was made possible by the generous support of the Global Down Syndrome Foundation, the Jerome Lejeune Foundation, Lumind RDS-Foundation, Matthews Foundation, Trisomie 21 France, Roche, Translational Therapeutics, IBRO, and the Down Syndrome International UK. Program material from this meeting can be found at http://www.t21rs.org/news-meetings.

References

dorno M, Sikandar S, Mitra SS, Kuo A, Nicolis Di Robilant B, et al: Usp16 contributes to somatic stem-cell defects in Down's syndrome. Nature 501:380-384 (2013).

-Ash JA, Velazquez R, Kelley CM, Powers BE, Ginsberg SD, et al: Maternal choline supplementation improves spatial mapping and increases basal forebrain cholinergic neuron number and size in aged Ts65Dn mice. Neurobiol Dis 70:32-42 (2014).
Bianchi DW: From prenatal genomic diagnosis to fetal personalized medicine: progress and challenges. Nature Med 18:1041-1051 (2012).

- Blehaut H, Mircher C, Ravel A, Conte M, de Portzamparc V, et al: Effect of leucovorin (folinic acid) on the developmental quotient of children with Down's syndrome (trisomy 21) and influence of thyroid status. PLoS One 5:e8394 (2010). 
Cossec JC, Lavaur J, Berman DE, Rivals I, Hoischen A, et al: Trisomy for synaptojanin 1 in Down syndrome is functionally linked to the enlargement of early endosomes. Hum Mol Genet 21:3156-3172 (2012).

Deidda G, Parrini M, Naskar S, Bozarth IF, Contestabile A, Cancedda L: Reversing excitatory GABAAR signaling restores synaptic plasticity and memory in a mouse model of Down syndrome. Nat Med 21:318-326 (2015).

de Sola S, de la Torre R, Sánchez-Benavides G, Benejam B, Cuenca-Royo A, et al: A new cognitive evaluation battery for Down syndrome and its relevance for clinical trials. Front Psychol 6:708 (2015).

Di Vona C, Bezdan D, Islam AB, Salichs E, LópezBigas N, et al: Chromatin-wide Profiling of DYRK1A Reveals a Role as a Gene-Specific RNA Polymerase II CTD Kinase. Mol Cell 57: 506-520 (2015).

-Gribble SM, Wiseman FK, Clayton S, Prigmore E, Langley E, et al: Massively parallel sequencing reveals the complex structure of an irradiated human chromosome on a mouse background in the tc1 model of Down syndrome. PLoS One 8: e60482 (2013).

Guedj F, Bianchi DW: Noninvasive prenatal testing creates an opportunity for antenatal treatment of Down syndrome. Prenat Diagn 33: 614-618 (2013).

-Guedj F, Sébrié C, Rivals I, Ledru A, Paly E, et al: Green tea polyphenols rescue of brain defects induced by overexpression of DYRK1A. PLoS One 4:e4606 (2009).

Guedj F, Bianchi DW, Delabar JM: Prenatal treatment of Down syndrome: a reality? Curr Opin Obstet Gynecol 26:92-103 (2014).

Guidi S, Stagni F, Bianchi P, Ciani E, Giacomini A, et al: Prenatal pharmacotherapy rescues brain development in a Down's syndrome mouse model. Brain 137:380-401 (2014).

-Gupta M, Dhanasekaran AR, Gardiner KJ: Mouse models of Down syndrome: gene content and consequences. Mamm Genome, E-pub ahead of print (2016).
Haydar TF, Reeves RH: Trisomy 21 and early brain development. Trends Neurosci 35:8191 (2012).

Hunter CL, Bachman D, Granholm AC: Minocycline prevents cholinergic loss in a mouse model of Down's syndrome. Ann Neurol 56: 675-688 (2004).

Marquer C, Laine J, Dauphinot L, Hanbouch L, Lemercier-Neuillet $C$, et al: Increasing membrane cholesterol of neurons in culture recapitulates Alzheimer's disease early phenotypes. Mol Neurodegener 9:60 (2014).

Moon J, Chen M, Gandhy SU, Strawderman M, Levitsky DA, et al: Perinatal choline supplementation improves cognitive functioning and emotion regulation in the Ts65Dn mouse model of Down syndrome. Behav Neurosci 124:346-361 (2010).

Ness S, Rafii M, Aisen P, Krams M, Silverman W, Manji H: Down's syndrome and Alzheimer's disease: towards secondary prevention. Nat Rev Drug Discov 11:655-656 (2012).

Noll C, Planque C, Ripoll C, Guedj F, Diez A, et al: DYRK1A, a novel determinant of the methionine-homocysteine cycle in different mouse models overexpressing this Downsyndrome-associated kinase. PLoS One 4:e7540 (2009).

O’Doherty A, Ruf S, Mulligan C, Hildreth V, Errington ML, et al: An aneuploid mouse strain carrying human chromosome 21 with Down syndrome phenotypes. Science 309:20332037 (2005).

Pujol J, del Hoyo L, Blanco-Hinojo L, de Sola S, Macià D, et al: Anomalous brain functional connectivity contributing to poor adaptive behavior in Down syndrome. Cortex 64:148156 (2015).

Salehi A, Delcroix JD, Belichenko PV, Zhan K, $\mathrm{Wu} \mathrm{C}$, et al: Increased App Expression in a mouse model of Down's syndrome disrupts NGF transport and causes cholinergic neuron degeneration. Neuron 51:29-42 (2006).
Sheehan R, Sinai A, Bass N, Blatchford P, Bohnen $\mathrm{I}$, et al: Dementia diagnostic criteria in Down syndrome. Int J Geriatr Psychiatry 30:857863 (2014).

Slonim DK, Koide K, Johnson KL, Tantravahi U, Cowan JM, et al: Functional genomic analysis of amniotic fluid cell-free mRNA suggests that oxidative stress is significant in Down syndrome fetuses. Proc Natl Acad Sci USA 106:9425-9429 (2009).

Souchet B, Guedj F, Sahún I, Duchon A, Daubigney F, et al: Excitation/inhibition balance and learning are modified by Dyrkla gene dosage. Neurobiol Dis 69:65-75 (2014).

Stagni F, Magistretti J, Guidi S, Ciani E, Mangano $\mathrm{C}$, et al: Pharmacotherapy with fluoxetine restores functional connectivity from the dentate gyrus to field CA3 in the Ts65Dn mouse model of Down syndrome. PLoS One 8: e61689 (2013).

Sun XL, He GQ, Song WH: BACE2, as a novel APP theta-secretase, is not responsible for the pathogenesis of Alzheimer's disease in Down syndrome. FASEB J 20:1369-1376 (2006).

Velazquez R, Ash JA, Powers BE, Kelley CM, Strawderman M, et al: Maternal choline supplementation improves spatial learning and adult hippocampal neurogenesis in the Ts65Dn mouse model of Down syndrome. Neurobiol Dis 58:92-101 (2013).

Visootsak J, Mahle WT, Kirshbom PM, Huddleston L, Caron-Besch M, et al: Neurodevelopmental outcomes in children with Down syndrome and congenital heart defects. Am J Med Genet A 155A:2688-2691 (2011).

-Wu Y, Deng Y, Zhang S, Luo Y, Cai F, et al: Amyloid- $\beta$ precursor protein facilitates the regulator of calcineurin 1-mediated apoptosis by downregulating proteasome subunit $\alpha$ type-5 and proteasome subunit $\beta$ type-7. Neurobiol Aging 36:169-177 (2015).

Zhang X, Wu Y, Duan X, Chen W, Zou H, et al: Upregulation of SET expression by BACE1 and its implications in Down syndrome. Mol Neurobiol 51:781-790 (2015). 